\title{
Neurohormonal signalling controls insulin sensitivity and specificity in $C$. elegans
}

Nicholas O. Burton ${ }^{1,2, \#}$, Vivek K. Dwivedi ${ }^{1}$, Kirk B. Burkhart ${ }^{1}$, Rebecca E. W. Kaplan ${ }^{3}$, L. Ryan Baugh ${ }^{3}$, and H. Robert Horvitz ${ }^{1 \#}$

${ }^{1}$ Howard Hughes Medical Institute, Department of Biology Massachusetts Institute of Technology, Cambridge, MA 02139, USA

${ }^{2}$ Centre for Trophoblast Research, Department of Physiology, Development and Neuroscience University of Cambridge, Cambridge, CB2 1HP, UK

${ }^{3}$ Department of Biology Duke University, Durham, NC 27708, USA

\# Correspondence: horvitz@mit.edu or nob20@cam.ac.uk 
Insulin and insulin-like growth factor signalling regulates a broad spectrum of growth and metabolic responses to a variety of internal and environmental stimuli. Such responses can be tailored to the environment so that changes in insulin signalling result in distinct physiological responses to different stimuli. For example, the inhibition of insulin-like signalling by osmotic stress or by starvation of $C$. elegans results in physiologically distinct states and patterns of gene expression. How does insulin-like signalling elicit different responses to different environmental stimuli? We report that neurohormonal signalling involving the $C$. elegans cytosolic sulfotransferase SSU-1 controls developmental arrest in response to osmotic stress but not to starvation; that SSU-1 functions in a single pair of sensory neurons to control signalling via the nuclear hormone receptor NHR-1; that signalling controlled by SSU-1 acts antagonistically to insulin-like signalling; and that the FOXO transcription factor DAF-16, a downstream effector of insulin-like signalling, enters the nucleus in response to osmotic stress but activates gene expression only if SSU-1 is active. We propose that neurohormonal signalling controlled by one or more cytosolic sulfotransferases similarly regulates the specificity of responses to changes in insulin signalling during periods of environmental stress in other organisms and that abnormalities in such sulfotransferasecontrolled neurohormonal signalling might contribute to human disorders that involve insulin signalling, such as obesity and type 2 diabetes. 
We previously reported that $C$. elegans arrests its development immediately after hatching in response to osmotic stress and that this developmental arrest is caused by the inhibition of insulin-like signalling and subsequent activation of the FOXO transcription factor DAF-16 ${ }^{1}$. Other studies showed that $C$. elegans also arrests its development immediately after hatching in response to starvation and that this developmental arrest is similarly caused by the inhibition of insulin-like signalling and activation of DAF-16 2 . Despite these similarities in the timing and regulation of developmental arrest in response to osmotic stress and starvation, we found that these two developmental arrests are physiologically distinct ${ }^{1}$. Specifically, (a) developmental arrest in response to osmotic stress results in animals that are immobile and do not respond to touch, whereas animals arrested in response to starvation remain mobile, and (b) most genes that exhibit changes in expression in animals arrested in response to osmotic stress do not exhibit changes in expression in animals arrested in response to starvation ${ }^{1}$. These results suggest that the inhibition of insulin-like signalling can elicit different physiological responses to starvation and osmotic stress. The mechanistic basis of these different responses is unknown.

To determine how the inhibition of insulin-like signalling can result in distinct states of arrested development in response to osmotic stress and starvation, we screened for mutants that failed to arrest development in response to osmotic stress (500 mM $\mathrm{NaCl}$ ). We identified a nonsense allele of the cytosolic sulfotransferase $s s u-1$ (n5888, W284Stop) that resulted in approximately $40 \%$ of animals failing to arrest 
development in response to $500 \mathrm{mM} \mathrm{NaCl}$ (Fig. 1a and 1b). Similarly, six independently isolated mutations in $s s u-1(f c 73, t m 1117, g k 266317, g k 747222$, $g k 876992, g k 319712$ ) caused animals to fail to arrest development in response to osmotic stress (Fig. 1b). SSU-1 is expressed in a single pair of sensory neurons, the ASJ neurons ${ }^{3}$. To determine if SSU-1 functions specifically in the ASJ sensory neurons to regulate developmental arrest in response to osmotic stress we expressed a rescuing transgene of $s s u-1(+)$ under the control of the ASJ-specific promoter trx-1 in $s S u-1(-)$ animals ${ }^{6}$. Expression of SSU-1 in the ASJ sensory neurons restored developmental arrest in response to osmotic stress (Fig. 1c), indicating that SSU-1 functions in the ASJ sensory neurons. We conclude that SSU-1 promotes developmental arrest in response to osmotic stress. SSU-1 is the only predicted cytosolic sulfotransferase encoded in the C. elegans genome ${ }^{3}$ and is most similar to the human cytosolic sulfotransferase SULT2A1, which sulfonates a variety of endogenous steroids, including dehydroepiandrosterone ${ }^{4}$. We propose that SSU-1 controls neurohormonal signalling by sulfonating signalling molecules in the ASJ sensory neurons to regulate developmental arrest in response to osmotic stress.

To test if SSU-1 is also required for developmental arrest in response to starvation, we starved wild-type, daf-16 and ssu-1 mutant animals for one week and assayed the percentage of animals with a divided M cell; $M$ cell division does not occur in animals that arrest development in response to starvation ${ }^{2}$. We found that $100 \%$ of M cells in both wild-type animals and ssu-1 mutants arrested cell division (Fig. 1d). By contrast, $8 \%$ of $\mathrm{M}$ cells in daf-16 mutants failed to arrest cell division in response 
to starvation (Fig. 1d), consistent with previous observations ${ }^{2}$. These results indicate that SSU-1 is required for developmental arrest in response to osmotic stress but is not required for developmental arrest in response to starvation.

In humans, the cytosolic sulfotransferase SULT2A1 sulfonates steroid hormones such as dehydroepiandrosterone (DHEA) and pregnenolone ${ }^{4}$. These hormones in turn regulate gene expression by activating nuclear hormone receptors ${ }^{4}$. We hypothesized that SSU-1 might similarly regulate the sulfonation of a hormone that in turn promotes developmental arrest in response to osmotic stress by controlling the transcriptional response to osmotic stress. To test if SSU-1 regulates gene expression in response to osmotic stress, we exposed wild-type and ssu-1 mutant embryos to either $50 \mathrm{mM}$ or $500 \mathrm{mM} \mathrm{NaCl}$ for 3 hours and quantified mRNA expression using RNA-Seq. We found that the expression of 434 genes was upregulated greater than 2-fold in response to osmotic stress and that SSU-1 function was required for the expression of 106 of these genes (Supplemental Table 1), including 20 of the 25 genes that exhibited a greater than 10 -fold increase in expression in response to osmotic stress $(\mathrm{p}<0.05)$ (Fig. 2a). For example, the genes that encode the superoxide dismutase sod-5 and the osmotic stress resistance protein $l e a-1^{6}$ exhibited a greater than 25 -fold increase in expression in response to osmotic stress and their increased expression in response to osmotic stress required SSU-1 (Fig. 2a). We confirmed that SSU-1 was required for the increased expression of sod-5 in response to osmotic stress using a GFP reporter (Figs. 2b and 2c). GFP was expressed broadly throughout the animal in response to osmotic 
stress, and this broad increase in expression required SSU-1 function in the ASJ sensory neurons (Figs. 2b and 2c).

To identify the transcription factor that functions downstream of SSU-1 to control the transcriptional response to osmotic stress we screened for additional mutants that 1) failed to arrest development in response to osmotic stress and 2) failed to express sod-5::gfp in response to osmotic stress. We identified five alleles of the nuclear hormone receptor $n h r-1$ (n6217, n6219, n6228, n6231, n6242) (Figs. 2d and 2e). In addition to failing to express sod-5::GFP in embryos arrested in response to osmotic stress, we found that $n h r-1$ mutants failed to express sod-5::GFP in dauer arrested animals (Fig. 2f). The loss of sod-5::gfp expression in dauer animals was tissue specifically rescued in animals that expressed a wild-type copy of $n h r-1$ in the intestine, using the ges- 1 promoter, neurons, using the $r a b-3$ promoter, and muscle, using the myo-3 promoter (Fig. 2f). However, we were unable to restore sod-5::gfp expression in $n h r-1$ mutant embryos using these three promoters or restore sod5::gfp expression using the endogenous $n h r-1$ promoter. We suspect that overexpression of $n h r-1$ in embryos might promote developmental arrest and prevent the recovery of transgenic animals. We conclude that NHR-1 functions cell autonomously to promote the expression of stress response genes, such as sod-5, in arrested animals.

We found that overexpression of $s s u-1$ in the ASJ sensory neurons was sufficient to drive the expression of sod-5::gfp even in the absence of osmotic stress (Fig. 2g). To 
test if the expression of sod-5::gfp in animals that overexpress $s s u-1$ requires NHR-1 we examined $n h r-1$ mutants that overexpressed $s s u-1$. We found that the overexpression of $s s u-1$ did not result in sod-5::gfp expression in $n h r-1$ mutants (Fig. $2 \mathrm{~g}$ ). These results indicate that NHR-1 is required for SSU-1 to drive the expression of sod-5 and suggest that SSU-1 and NHR-1 function in the same pathway. We propose that SSU-1 sulfonates the ligand for NHR-1 in the ASJ sensory neurons and that this ligand then signals to diverse tissues to activate NHR-1 and drive the transcriptional response to osmotic stress and developmental arrest.

Osmotic stress causes developmental arrest of $C$. elegans by inhibiting insulin-like signalling, which results in the activation of the FOXO transcription factor DAF-16 ${ }^{1}$. To determine if DAF-16 and SSU-1 regulate the expression of the same or different downstream target genes we exposed wild-type and daf-16 mutant embryos to 50 $\mathrm{mM}$ or $500 \mathrm{mM} \mathrm{NaCl}$ and quantified mRNA expression by RNA-seq. We found that 161 genes both exhibited a greater than 2-fold increase in expression in response to osmotic stress and were dependent on DAF-16 for their induction (Fig. 3a-b and Supplemental Table 2). Notably, all of the top 25 genes that exhibit the largest DAF16 dependent increase in expression in response to osmotic stress also require SSU1 for their expression, and all of the top 25 genes that exhibit the largest SSU-1 dependent increase in expression in response to osmotic stress also require DAF-16 for their expression (Supplemental Table 2). In total, of the 161 genes regulated by DAF-16 in response to osmotic stress, 64 were also regulated by SSU-1 (Fig. 3c). By contrast, genes such as gpdh-1, which exhibits an approximately 50 -fold increase in 
response to osmotic stress, did not require either SSU-1 or DAF-16 for their expression (Fig. 2a and 3a). These results indicate that most of the genes that exhibit the largest increase in expression in response to osmotic stress require both SSU-1 and DAF-16 for their expression.

Mutations in the insulin-like receptor gene $d a f-2$ result in animals that are more likely to arrest development in response to osmotic stress than wild-type animals ${ }^{1}$. To examine potential interactions between $s s u-1$ and $d a f-2$ we constructed double mutant animals harboring mutations in both $d a f-2$ and $s s u-1$ and exposed wild-type, daf-2, ssu-1, and daf-2; ssu-1 double mutant embryos to mild osmotic stress $(300 \mathrm{mM}$ $\mathrm{NaCl}$ ) and strong osmotic stress (500 mM NaCl). Consistent with our previous findings, we found that nearly $100 \%$ of daf-2 mutant embryos arrested development at $300 \mathrm{mM} \mathrm{NaCl}$ (Fig. 3d). However, none of the wild-type, ssu-1, or daf-2; ssu-1 double mutant animals arrested development at $300 \mathrm{mM} \mathrm{NaCl}$ (Fig. 3d). In addition, we found that approximately $30 \%$ of $s s u-1$ and $d a f-2$; ssu-1 double mutant animals failed to arrest development even at $500 \mathrm{mM} \mathrm{NaCl}$ (Fig. 3e). These results indicate that the loss of SSU-1 function can suppress the effects of reduced insulin-like signalling in response to osmotic stress.

Insulin-like signalling inhibits the activation of the FOXO transcription factor DAF16 by sequestering it in the cytoplasm ${ }^{7}$. The loss of insulin-like signalling in response to osmotic stress causes developmental arrest because DAF-16 is no longer sequestered in the cytoplasm and can translocate into the nucleus ${ }^{1}$. We 
hypothesized that SSU-1 signalling might promote the translocation of DAF-16 into the nucleus in response to osmotic stress in parallel to insulin-like signalling. To test this hypothesis, we examined wild-type and $s s u-1$ mutant animals that expressed a GFP-tagged copy of DAF-16 and assayed DAF-16 translocation to the nucleus in response to osmotic stress. We found that SSU-1 was not required for DAF-16 translocation into the nucleus in response to osmotic stress (Fig. 3f and Supplemental Fig. 1). This observation suggests that signalling via SSU-1 functions in parallel to insulin-like signalling and DAF-16 translocation into the nucleus to regulate development and gene expression in response to osmotic stress.

We previously found that increases in the levels of glycerol, an osmolyte that protects animals from the effects of osmotic stress, can protect C. elegans from developmental arrest in response to osmotic stress ${ }^{1}$. We hypothesized that the loss of SSU-1 or DAF-16 might similarly result in increased production of glycerol to protect animals from entering developmental arrest in response to osmotic stress. To test this hypothesis we profiled 137 polar metabolites and 1,069 lipid metabolites in wild-type, ssu-1, and $d a f-16$ mutant embryos by mass spectrometry (Supplemental Table 3). Four polar molecules and four lipid molecules were changed in both ssu-1 and daf-16 mutant embryos (Figs. $4 \mathrm{a}$ and $4 \mathrm{~b}$ ). We did not observe an increase in the levels of glycerol, indicating that the loss of SSU-1 and DAF-16 does not protect animals from developmental arrest in response to osmotic stress by increasing the production of glycerol. In addition, we found that none of the metabolites we profiled exhibited a greater than 2 -fold change in abundance, 
suggesting that under normal osmotic conditions the loss of either SSU-1 or DAF-16 has minimal effects on metabolism.

Alternatively, SSU-1 and DAF-16 might regulate the production of glycerol specifically in response to osmotic stress, such that in response to osmotic stress ssu-1 and daf-16 mutants produce more glycerol, which protects animals from developmental arrest. To determine how SSU-1 and DAF-16 regulate metabolism in response to osmotic stress we profiled polar and lipid metabolites in embryos exposed to $300 \mathrm{mM} \mathrm{NaCl}$. We identified 6 polar metabolites that increased in abundance more than 2 -fold $(\mathrm{p}<0.01)$ in wild-type animals in response to osmotic stress, including glycerol, and 12 polar metabolites that decreased in abundance more than 2 -fold ( $\mathrm{p}<0.01$ ) in wild-type animals in response to osmotic stress, including several TCA cycle intermediates (Supplemental Table 2). Of these 18 polar metabolites that changed in abundance in response to osmotic stress none were regulated by both SSU-1 and DAF-16 (Supplemental Table 2). In addition, we identified 63 lipid metabolites that exhibited a greater than 2 -fold decrease in abundance in response to osmotic stress and 85 lipid metabolites that exhibited a greater than 2-fold increase in abundance in response to osmotic stress. Of these 148 lipid metabolites that change in abundance, six were regulated by both SSU-1 and DAF-16 and all six were lysophosphatidylcholines (Fig. 4c). Specifically, we identified six lysophosphatidylcholines that exhibited between two and eight fold increases in abundance in response to osmotic stress and this increase in abundance required both SSU-1 and DAF-16 (Fig. 4c). These findings show that embryonic 
metabolism is affected by osmotic stress and suggest that changes in

lysophosphatidylcholine metabolism are involved in the response to osmotic stress.

In short, we have identified a neurohormonal signalling pathway that regulates insulin sensitivity and specificity in C. elegans. Specifically, we found that neurohormonal signalling via SSU-1 was required for the specificity of developmental arrest in response to osmotic stress, but not starvation, and that the loss of neurohormonal signalling via SSU-1 could suppress the enhanced sensitivity of insulin-like receptor (daf-2) mutants to osmotic stress. The finding that SSU-1 is required to control the expression of DAF-16 target genes in response to osmotic stress sheds light on how the inhibition of insulin-like signalling by both osmotic stress and starvation can cause different states of arrested development. We propose that SSU-1 functions in parallel to the FOXO transcription factor DAF-16 to promote a transcriptional response to osmotic stress, the production of lysophophatidylcholines, and developmental arrest in response to osmotic stress (Fig. 4d). We speculate that the regulation of development and modification of insulin signalling specificity by cytosolic sulfotransferases and nuclear hormone receptors is a conserved process and that studies of how cytosolic sulfotransferases affect metabolism and insulin specificity will provide important insights into disorders that involve insulin signalling, including obesity and type-2 diabetes. 


\section{Materials and Methods}

Strains. C. elegans strains were cultured as described ${ }^{8}$ and maintained at $20^{\circ} \mathrm{C}$ unless noted otherwise. The Bristol strain N2 was the wild-type strain. Mutations used were:

LGI: $d a f-16(m 26), d a f-16(m u 86)$

LGIII: $d a f-2(e 1370)$

LGIV: ssu-1(fc73), ssu-1(n5883), ssu-1(tm1117), ssu-1(gk266317), ssu-1(gk747222), ssu-1(gk876992), ssu-1(gk319712), zIs356 [Pdaf-16::daf-16a/b-gfp; rol-6]

LGX: $n h r-1(n 6217), n h r-1(n 6219), n h r-1(n 6228), n h r-1(n 6231), n h r-1(n 6242)$

Unknown linkage: wuIs57 [sod-5p::GFP, rol-6(su1006)]

Extrachromosomal arrays: $n E x 2685$ [Ptrx-1::ssu-1::mCherry::unc-54 3'UTR; Punc122::GFP], nEx2722 [Prab-3::nhr-1::tbb-2 3'UTR], nEx2720 [Pmyo-3::nhr1::tbb-2 3'UTR], nEx2719[Pges-1::nhr-1::tbb-2 3'UTR]

M cell division in response to starvation

Analyses of M cell division were performed as described ${ }^{2}$.

\section{DAF-16::GFP localization:}

Embryos were placed onto Petri plates containing $500 \mathrm{mM} \mathrm{NaCl}$ in NGM agar seeded with E. coli OP50 for $5 \mathrm{hrs}$. Confocal microscopy was performed using a Zeiss LSM 800 instrument. The resulting images were prepared using ImageJ software (National Institutes of Health). Image acquisition settings were calibrated to 
minimize the number of saturated pixels and were kept constant throughout the experiment.

\section{Assay for developmental arrest}

Approximately 200 developing eggs from mothers grown at $50 \mathrm{mM} \mathrm{NaCl}$ (unless otherwise noted) were collected and placed on standard NGM Petri plates containing varying concentrations of $\mathrm{NaCl}$ for $48 \mathrm{hrs}$. After $48 \mathrm{hrs}$, animals that remained immobile and were not feeding were scored as arrested. Mobile animals that were feeding were scored as developing. Percent failing to arrest was defined as the percent of animals mobile and feeding (unlike animals normally arrested in response to osmotic stress) and includes L1-stage larvae.

\section{Mutagenesis screen for animals that fail to arrest development}

Approximately 20,000 L4 stage wild-type animals were incubated with $20 \mu \mathrm{l}$ of ethyl methanesulfonate (Sigma) in $4 \mathrm{~mL}$ of M9 for $4 \mathrm{hrs}$ at $20^{\circ} \mathrm{C}$. F3 generation embryos were placed onto Petri plates containing $500 \mathrm{mM} \mathrm{NaCl}$ in NGM agar and screened for mutants that hatched and were mobile.

\section{Metabolite preparation and quantification}

Approximately $100 \mu \mathrm{l}$ of embryos were placed on standard NGM agar plates. After 3

hrs embryos were collected in M9, pelleted, and frozen. $100 \mu \mathrm{l}$ of frozen embryos were resuspended in $600 \mu \mathrm{l}$ methanol and lysed using a BeadBug microtube homogenizer (Sigma) and $0.5 \mathrm{~mm}$ Zirconium beads. After lysis, $300 \mu \mathrm{l}$ of water and 
$400 \mu \mathrm{l}$ of cholorform was added to each sample and samples were then vortexed for

$1 \mathrm{~min}$ at $4^{\circ} \mathrm{C}$ and centrifuged for $10 \mathrm{~min}$ at $15000 \mathrm{~g}$ at $4^{\circ} \mathrm{C}$. After centrifugation the polar and lipid layers were separated and dried using a SpeedVac concentrator. Liquid chromatography and mass spectrometry were performed as described ${ }^{9}$.

\section{$\underline{R N A-S e q}$}

Wild-type, $s s u$-1(fc73), and $d a f-16(m 26)$ embryos were placed on standard NGM plates. After 3 hrs embryos were collected in M9, lysed using a BeadBug microtube homogenizer (Sigma) and $0.5 \mathrm{~mm}$ Zirconium beads (Sigma), and RNA was extracted using the RNeasy Mini kit (QIAGEN). RNA integrity and concentration were checked using a Fragment Analyzer (Advanced Analytical), and libraries were prepared using a Illumina NeoPrep RNAseq kit. Libraries were quantified using the Fragment Analyzer (Advanced Analytical) and qPCR before being loaded for paired-end sequencing using the Illumina NextSeq500. 75-nucleotide paired-end sequencing reads were mapped against the $C$. elegans genome assembly ce10 using RSEM v. 1.2.15, with Bowtie v. 1.0.1 for read alignment (flags --paired-end -p 6 --bowtiechunkmbs 1024 --forward-prob 0, for strand-specific libraries) ${ }^{10-11}$. Expected read counts per gene were retrieved and, after rounding them to the nearest integer, used to perform differential gene expression with DESeq2 in the R v. 3.2.3 statistical environment ${ }^{12-13}$. Sequencing library size factors were estimated for each library; to account for differences in sequencing depth and complexity among libraries, as well as gene-specific count dispersion parameters (reflecting the relationship between the variance in a given gene's counts and that gene's mean expression across 
samples). Differences in gene expression between conditions (expressed as log2transformed fold-changes in expression levels) were estimated under a general linear model (GLM) framework fitted on the read counts. In this model, read counts of each gene in each sample were modeled under a negative binomial distribution, based on the fitted mean of the counts and aforementioned dispersion parameters. Differential expression significance was assessed using a Wald test on the fitted count data (all these steps were performed using the DESeq0 function in DESeq2) ${ }^{13}$. The DESeq2 algorithm also proposes two filtering steps to remove noisy outliers, one named "independent filtering", based on the mean of normalized count, and a second one, based on the Cook's distance, which assess how much the fit of the distribution would be impacted by removing an individual sample ${ }^{13-14}$. The results were the same in the presence or absence of these two filtering steps for the N2 vs. ssu-1 comparisons. For the N2 vs. daf-16 experiment, these two filtering steps were turned off for the purpose of identifying differentially-expressed genes, out of a concern that they would lead to the undue elimination of noisy, yet real differentially-expressed genes in that samples series. P-values were adjusted for multiple-comparison testing using the Benjamini-Hochberg procedure ${ }^{14-15}$ and genes with adjusted $\mathrm{p}$-value $<0.05$ and absolute fold-change greater than 2 were used for downstream analyses. To compare different mutants against wildtype, subsets of genes meeting the above-mentioned differential expression criteria in each mutant-wildtype comparison were intersected and enumerated Gene identifiers were retrieved from Wormbase version WS235. Hierarchical clustering 
and visualization of the data was performed in Spotfire (Tibco), using a complete linkage function and correlation as a distance metric both for genes and samples.

\section{Psod-5::GFP images}

Embryos were placed onto Petri plates containing either $50 \mathrm{mM}$ or $500 \mathrm{mM} \mathrm{NaCl}$ in NGM agar. Arrested L1 stage animals at $500 \mathrm{mM} \mathrm{NaCl}$ were imaged after $24 \mathrm{hrs}$ (Fig.

$2 \mathrm{~b}$ and $2 \mathrm{c}$ ). Early L1 stage animals at $50 \mathrm{mM} \mathrm{NaCl}$ were imaged after $5 \mathrm{hrs}$ to control for staging (Fig. 2a and 2g). Dauer animals were collected 7 days after starvation on plates containing $50 \mathrm{mM} \mathrm{NaCl}$ (Fig. 2f). Confocal microscopy was performed using a Zeiss LSM 800 instrument for Figures $2 \mathrm{~b}$ and 2c. Confocal microscopy was performed using a Leica SP8 instrument for Figures $2 \mathrm{f}$ and $2 \mathrm{~g}$. The resulting images were prepared using ImageJ software (National Institutes of Health). Image acquisition settings were calibrated to minimize the number of saturated pixels and were kept constant throughout each experiment.

\section{Cloning of Ptrx-1::SSU-1::mCherry}

A synthetic ssu-1 DNA fragment with synthetic introns replacing endogenous introns (endogenous introns are repetitive and could not be synthesized) was obtained from Integrated DNA Technologies using their custom gene synthesis service. The ssu-1 fragment was amplified with a 3' 5xGly linker using appropriate primers. The pJDM169 vector containing $1.1 \mathrm{~kb}$ of the $\operatorname{tr} x-1$ promoter sequence upstream of the trx-1 start codon, mCherry and an unc-54 3'UTR was obtained from J. Meisel (Meisel and Kim, 2014). The ssu-1 DNA fragment with 5xGly linker was 
cloned into the pJDM169 using Infusion HD (Clontech) cloning to generate the plasmid pVD100 that contains Ptrx-1::ssu-1(cDNA) - 5xGly- mCherry::unc-54 3'UTR.

\section{Cloning of nhr-1 rescuing transgenes}

R09G11.2c was amplified from C. elegans wild-type cDNA and used in all vectors generated for tissue-specific expression of $n h r-1$ cDNA. Infusion HD (Clontech) cloning was used to clone individual promoter fragments with $n h r-1$ cDNA, $5 x$ Gly linker, mCherry and $t b b-23^{\prime} U T R$ to generate plasmids containing the generic sequence Promoter::nhr-1 cDNA - 5xGly - mCherry::tbb-2 3'UTR. The promoter fragments of the genes ges-1 (in pVD104), unc-54 (in pVD105), rab-3 (in pVD106) and $d p y-7$ (in pVD107) used for tissue-specific expression of $n h r-1$ cDNA contain 2.9, $1.9,1.4$ and $1.3 \mathrm{~kb}$, respectively, of sequence upstream of each of these genes' start codons.

\section{Statistics and Reproducibility}

Two-tail t-tests were used to compare all samples that reflect percentages of populations or populations of animals. No statistical method was used to predetermine sample size. The experiments were not randomized. The investigators were not blinded to allocation during experiments and outcome assessment.

Statistics source data available in Supplemental Table 4.

Acknowledgments: We thank David Gems and the Caenorhabditis Genetic Center, which is funded by the NIH National Center for Research Resources (NCRR), for 
providing strains; N. An for strain management; and A. Doi, and A. Corrionero for

helpful discussions. HRH, VD, KB, and NOB were supported by NIH grant GM024663

and NOB was supported by NSF grant 1122374. VD was a Howard Hughes Medical

Institute International Student Research fellow. LRB and REWK were supported by NIH grant GM117408.

\section{References:}

1. Burton, N.O., Furuta, T., Webster, A.K., Kaplan, R.E.W., Baugh, L.R., Arur, S., and Horvitz, H.R. (2017). Insulin-like signalling to the maternal germline controls progeny response to osmotic stress. Nat Cell Biol 19, 252-257.

2. Baugh, L.R., and Sternberg, P.W. (2006). DAF-16/FOXO regulates transcription of cki-1/Cip/Kip and repression of lin-4 during C. elegans L1 arrest. Curr Biol 16, 780785.

3. Carroll, B.T., Dubyak, G.R., Sedensky, M.M., and Morgan, P.G. (2006). Sulfated signal from ASJ sensory neurons modulates stomatin-dependent coordination in Caenorhabditis elegans. J Biol Chem 281, 35989-35996.

4. Coughtrie, M.W.H. (2016). Function and organization of the human cytosolic sulfotransferase (SULT) family. Chem Biol Interact 259, 2-7.

5. Miranda-Vizuete, A., Fierro Gonzalez, J.C., Gahmon, G., Burghoorn, J., Navas, P., and

Swoboda, P. (2006). Lifespan decrease in a Caenorhabditis elegans mutant lacking TRX-1, a thioredoxin expressed in ASJ sensory neurons. FEBS Lett 580, 484-490. 
6. Gal, T. Z., Glazer, I. \& Koltai, H (2004).. An LEA group 3 family member is involved in survival of C. elegans during exposure to stress. FEBS Lett 577, 21-26.

7. Murphy, C.T., and Hu, P.J. (2013). Insulin/insulin-like growth factor signaling in $C$. elegans. WormBook 1-43.

8. Brenner, S. (1974). The genetics of Caenorhabditis elegans. Genetics 77, 71-94

9. Birsoy, K. et al. (2015). An essential role of the mitochondrial electron transport chain in cell proliferation is to enable aspartate synthesis. Cell 162, 540-551

10. Langmead B., Trapnell C, Pop M, Salzberg SL. Ultrafast and memory-efficient alignment of short DNA sequences to the human genome. Genome Biol. 2009;10(3):R25. doi: 10.1186/gb-2009-10-3-r25. Epub 2009 Mar 4.

11. Li B, Dewey CN. RSEM: accurate transcript quantification from RNA-Seq data with or without a reference genome. BMC Bioinformatics. 2011 Aug 4;12:323. doi: 10.1186/1471-2105-12-323.

12. Anders, S., Huber, W. Differential expression analysis for sequence count data. Genome Biol. 2010;11(10):R106. doi: 10.1186/gb-2010-11-10-r106. Epub 2010 Oct 27.

13. Michael I Love, Wolfgang Huber, Simon Anders: Moderated estimation of fold change and dispersion for RNA-seq data with DESeq2. Genome Biology 2014, $15: 550$.

14. Cook, R. D. 1977. “Detection of Influential Observation in Linear Regression.” Technometrics, 1977 (February), 19(1):15-18 
15. Benjamini, Y., Hochberg, Y. Controlling the false discovery rate: a practical and powerful approach to multiple testing. Journal of the Royal Statistical Society, Series B. 57 (1): 289-300.

16. Meisel, J. D. \& Kim, D. H. Inhibition of Lithium-Sensitive Phosphatase BPNT-1 Causes Selective Neuronal Dysfunction in C. elegans. Curr Biol 26, 1922-1928 (2016).

\section{Figure Legends}

Fig. 1. The cytosolic sulfotransferase SSU-1 functions in the ASJ sensory neurons to regulate developmental arrest in response to osmotic stress. (a) Schematic of ssu-1 mutations that cause defects in developmental arrest in response to osmotic stress. Scale bar $100 \mathrm{bp}$ (b) Percent of ssu-1 mutants failing to arrest development in response to $500 \mathrm{mM} \mathrm{NaCl} . \mathrm{n}=3$ experiments represent greater than 100 animals. Error bars, s.d. (c) Number of wild-type, ssu-1(fc73), and ssu-1(fc73); ssu-1(+) animals failing to arrest development in response to osmotic stress. The $\operatorname{tr} x-1$ promoter was used to drive ASJ cell specific expression of $s s u-1$. Animals also contained wuIs57 [pPD95.77 Psod-5::GFP, rol-6(su1006)] which drives GFP expression in response to osmotic stress. $n=100$ animals (d) Percent of wild-type, daf-16(mu86), and ssu-1(fc73) animals with a divided M cell after 1 week of starvation. $\mathrm{n}>200$ animals. Error bars, s.e.m. ${ }^{*} \mathrm{p}<0.05,{ }^{* *} \mathrm{p}<0.01,{ }^{* * *} \mathrm{p}<0.001$ 
bioRxiv preprint doi: https://doi.org/10.1101/229393; this version posted December 5, 2017. The copyright holder for this preprint (which was not certified by peer review) is the author/funder, who has granted bioRxiv a license to display the preprint in perpetuity. It is made available under aCC-BY-NC-ND 4.0 International license.

a

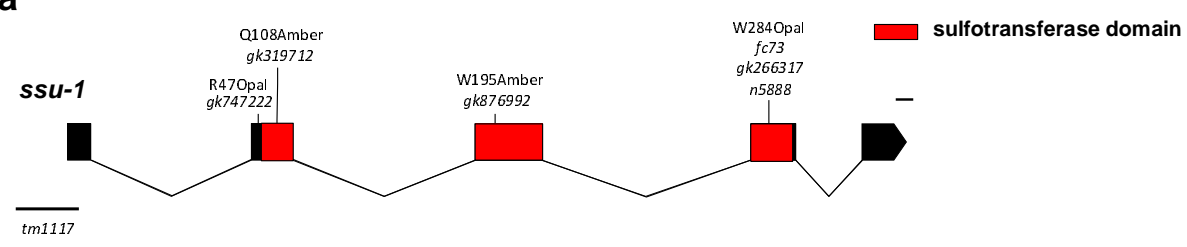

b

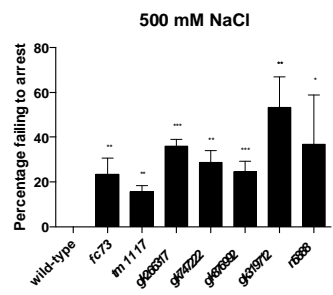

C

\begin{tabular}{cc}
\hline genotype* & $\begin{array}{c}\text { Number } \\
\text { failing to } \\
\text { arrest }\end{array}$ \\
\hline wild-type & 0 \\
ssu-1 & 42 \\
$\begin{array}{c}\text { SSU-1; } \\
\text { pASU::SSu-1 }\end{array}$ & 0 \\
\hline "strains \\
contained \\
PSod-5::GFP
\end{tabular}

d

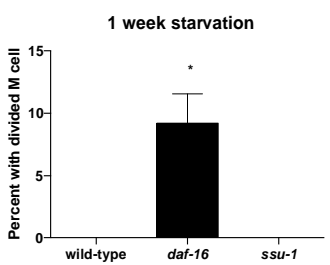


Fig. 2. The nuclear hormone receptor NHR-1 is required for SSU-1 to promote the transcriptional response to osmotic stress (a) Average fold change mRNA expression in wild-type and $s s u-1(f c 73)$ mutant animals at $500 \mathrm{mM} \mathrm{NaCl}$ when compared to $50 \mathrm{mM} \mathrm{NaCl}$ measured by RNA-Seq (FPKM $500 \mathrm{mM} \mathrm{NaCl/FPKM} 50 \mathrm{mM}$ $\mathrm{NaCl}+1$ ). Shown are the 25 genes that exhibited the greatest increase in expression in wild-type animals at $500 \mathrm{mM} \mathrm{NaCl}$ vs. $50 \mathrm{mM} \mathrm{NaCl} . \mathrm{n}=3$. Error bars s.d. (b) Confocal and differential interference contrast (DIC) images showing Psod-5::gfp expression in wild-type and $s s u-1(f c 73)$ mutants exposed to $500 \mathrm{mM} \mathrm{NaCl}$ for $24 \mathrm{hrs}$. Scale bars, $100 \mu \mathrm{m}$. (c) Confocal and DIC images of Psod-5::GFP expression in wildtype, ssu-1(fc73), and ssu-1(fc73); ssu-1(+) mutants exposed to $500 \mathrm{mM} \mathrm{NaCl}$ for 24 hrs. The trx-1 promoter was used to drive ASJ cell-specific expression of SSU-1. Scale bars, $100 \mu \mathrm{m}$. (d) Schematic of $n h r-1$ mutations that cause defects in developmental arrest in response to osmotic stress. Scale bar $100 \mathrm{bp}(\mathrm{e})$ Percent of $n h r-1$ mutants failing to arrest development in response to $500 \mathrm{mM} \mathrm{NaCl} . \mathrm{n}=3$ experiments represent greater than 100 animals. Error bars, s.d. (f) Representative confocal and DIC images of Psod-5::GFP expression in wild-type and $n h r-1(n 6242)$ mutant dauers. The ges-1 promoter was used to drive intestine specific expression, the $r a b-3$ promoter was used to drive neuron specific expression, and the myo-3 promoter was used to drive muscle specific expression. Scale bar $100 \mu \mathrm{m}$. (f) Representative confocal and DIC images of Psod-5::GFP expression in wild-type and $n h r-1(n 6242)$ L1 stage animals. The trx-1 promoter was used to drive ASJ cell-specific expression of SSU-1. Animals expressed GFP specifically in coelomocytes under the control of the unc-122 promoter as a coinjection marker. Percentages reflect the percentage of 
bioRxiv preprint doi: https://doi.org/10.1101/229393; this version posted December 5, 2017. The copyright holder for this preprint (which was not certified by peer review) is the author/funder, who has granted bioRxiv a license to display the preprint in perpetuity. It is made available under aCC-BY-NC-ND 4.0 International license.

animals that express GFP similar to the representative images. Scale bar $100 \mu \mathrm{m} * \mathrm{p}$ $<0.05,{ }^{* *} \mathrm{p}<0.01,{ }^{* * *} \mathrm{p}<0.001$ 
bioRxiv preprint doi: https://doi.org/10.1101/229393; this version posted December 5, 2017. The copyright holder for this preprint (which was not certified by peer review) is the author/funder, who has granted bioRxiv a license to display the preprint in perpetuity. It is made available under aCC-BY-NC-ND 4.0 International license.

a

C

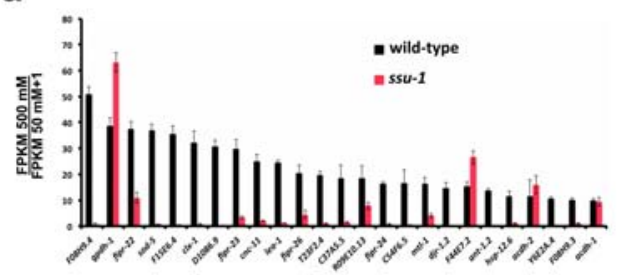

b

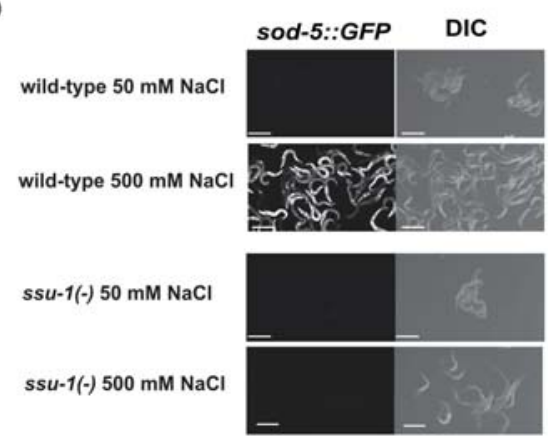
d

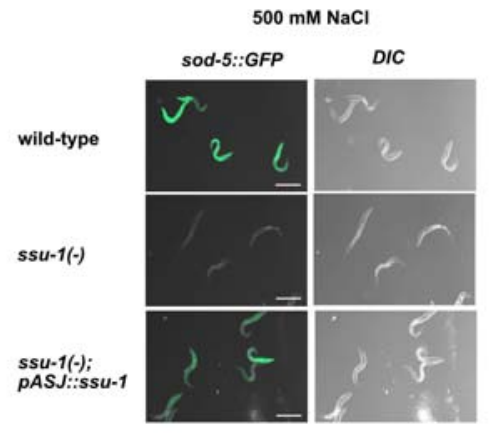

e

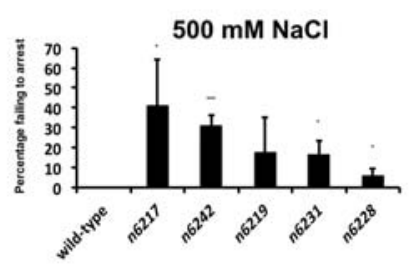

f

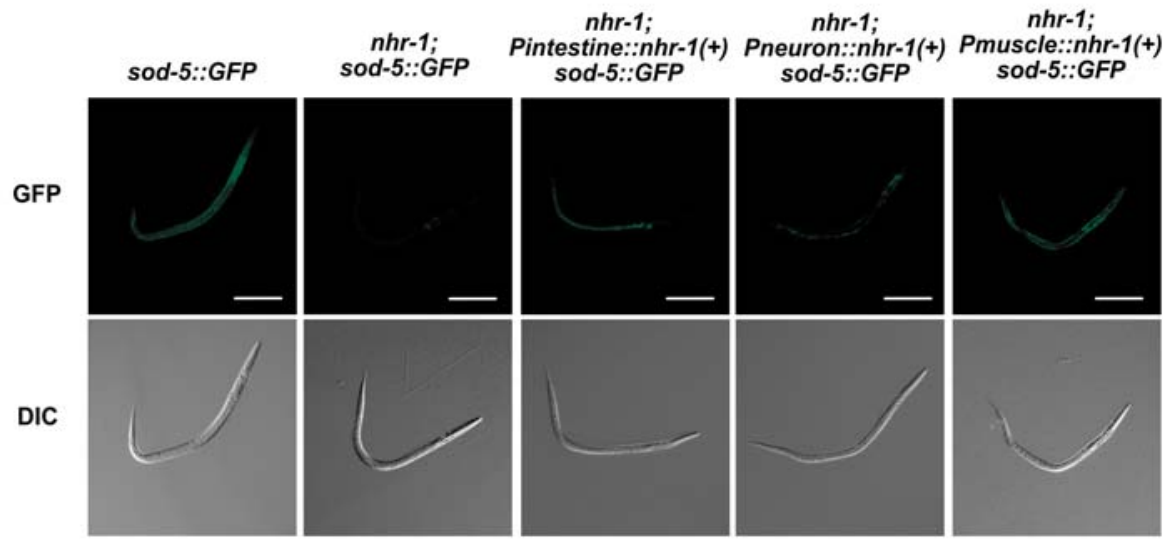

g

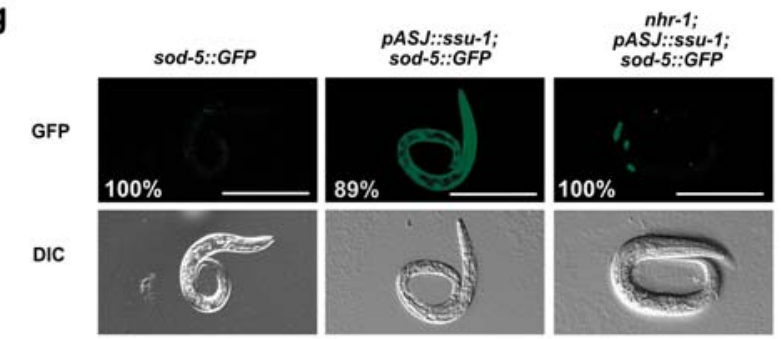


Fig. 3 SSU-1 and insulin-like signalling via the FOXO transcription factor DAF-16 function in parallel to regulate gene expression and developmental arrest in response to osmotic stress. (a) Average fold change mRNA expression in wild-type and $d a f-16(\mathrm{~m} 26)$ mutant animals at $500 \mathrm{mM} \mathrm{NaCl}$ when compared to $50 \mathrm{mM} \mathrm{NaCl}$ measured by RNA-Seq (FPKM $500 \mathrm{mM} \mathrm{NaCl}$ /FPKM $50 \mathrm{mM} \mathrm{NaCl}+1$ ). Shown are the 25 genes that exhibited the greatest increase in expression in wild-type animals at $500 \mathrm{mM} \mathrm{NaCl}$ vs. $50 \mathrm{mM} \mathrm{NaCl} . \mathrm{n}=3$. Error bars s.d. (b) Comparison of the 20 genes exhibiting the largest SSU-1 and DAF-16 dependent increases in expression in response to $500 \mathrm{mM} \mathrm{NaCl}$. (c) Venn diagram of genes with a greater than 2-fold increase in RNA expression in response to osmotic stress and dependent on SSU-1 (blue) and/or DAF-16 (yellow) (d) Percent of wild-type, daf-2(e1370) and ssu1(fc73) mutants failing to arrest development in response to $300 \mathrm{mM} \mathrm{NaCl} . \mathrm{n}>100$ animals, error bars s.d. (e) Percent of wild-type, daf-2(e1370) and ssu-1(fc73) mutants failing to arrest development in response to $500 \mathrm{mM} \mathrm{NaCl}$. $\mathrm{n}>100$ animals. Error bars, s.d. (f) Representative confocal images of DAF-16::GFP localization after 5 hrs of exposure to osmotic stress ( $500 \mathrm{mM} \mathrm{NaCl}$ ) in the wildtype and $s s u-1$ (fc 73 ) mutants. Scale bars, $10 \mu \mathrm{m} .{ }^{* * *} \mathrm{p}<0.001$ 
bioRxiv preprint doi: https://doi.org/10.1101/229393; this version posted December 5, 2017. The copyright holder for this preprint (which was not certified by peer review) is the author/funder, who has granted bioRxiv a license to display the preprint in perpetuity. It is made available under aCC-BY-NC-ND 4.0 International license.

a

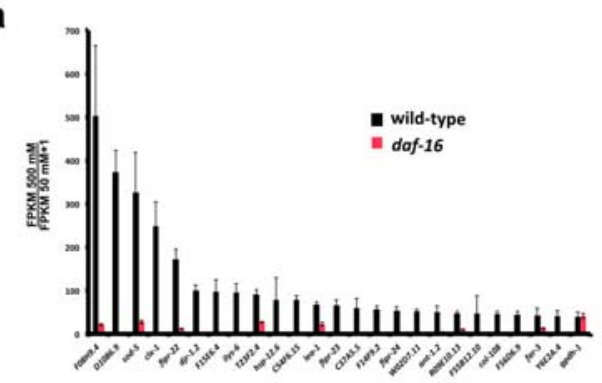

C

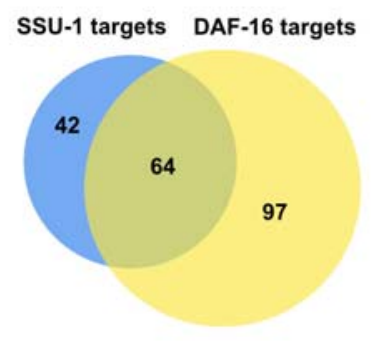

e

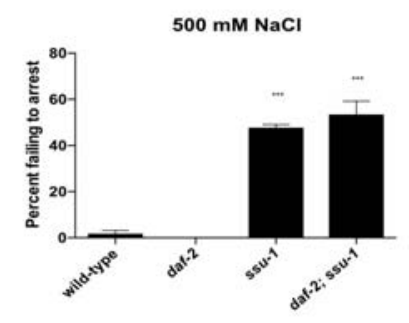

b

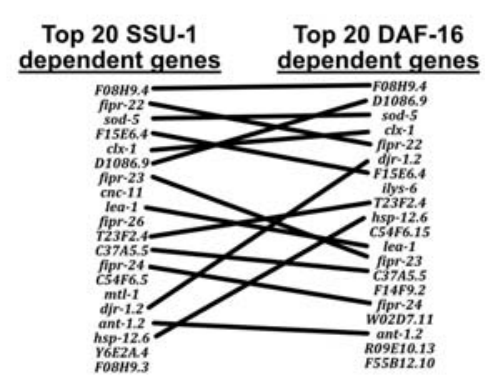

d

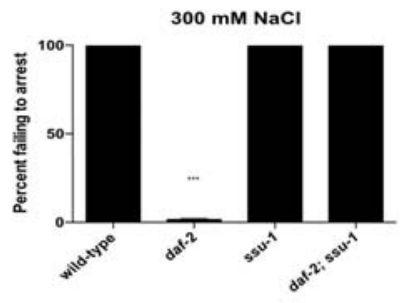

f

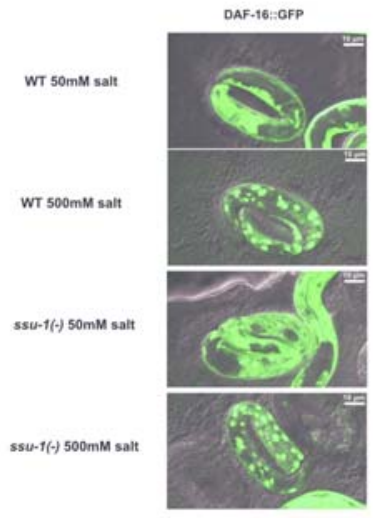


Fig. 4 SSU-1 and DAF-16 regulate lysophosphatidylcholine levels in response to osmotic stress (a) Relative levels of polar metabolites exhibiting a statistically significant $(\mathrm{p}<0.01)$ change in levels in ssu-1(fc73) and daf-16(m26) mutant embryos. Metabolites were normalized to the levels of histidine. $n=3$. Error bars, s.d. (c) Relative levels of lipid metabolites exhibiting a statistically significant ( $p<$ $0.01)$ change in levels in both $s s u-1(f c 73)$ and $d a f-16(\mathrm{~m} 26)$ mutant embryos. Metabolites were normalized to the levels of total lipid. $n=3$. Error bars, s.d. PC phosphatidylcholine; AcCa Acylcarnitine; PE phosphatidylethanolamine; TG triglyceride. (c) Relative levels of lysophosphatidylcholine (LPC) metabolites in wild-type, ssu-1(fc73) and $d a f-16(m 26)$ mutant embryos. Metabolites shown exhibited a statistically significant $(\mathrm{p}<0.01)$ and greater than 2 -fold increase in abundance in response to osmotic stress ( $300 \mathrm{mM} \mathrm{NaCl}$ ) and required both SSU-1 and DAF-16. Metabolites were normalized to the levels of total lipid. $n=3$. Error bars, s.d (d) Model for how C. elegans regulates metabolism and development in response to osmotic stress. See text for details. 
bioRxiv preprint doi: https://doi.org/10.1101/229393; this version posted December 5, 2017. The copyright holder for this preprint (which was not certified by peer review) is the author/funder, who has granted bioRxiv a license to display the preprint in perpetuity. It is made available under aCC-BY-NC-ND 4.0 International license.

a

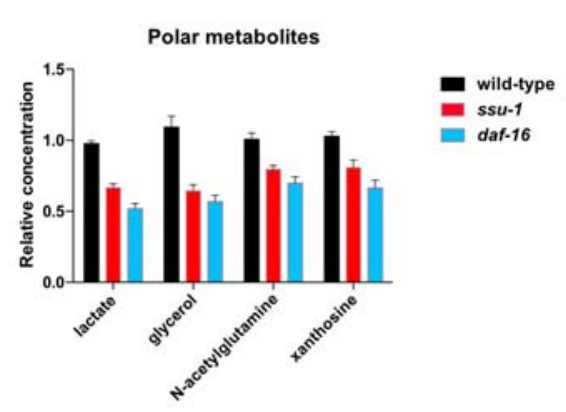

C

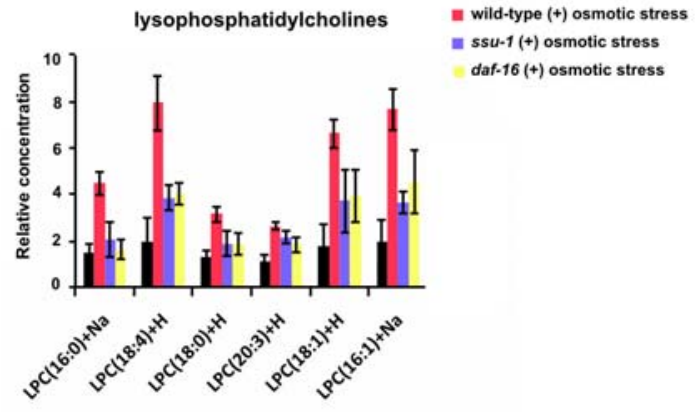

b

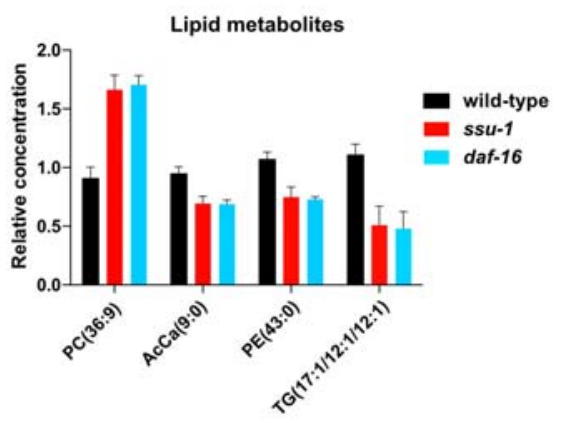

d

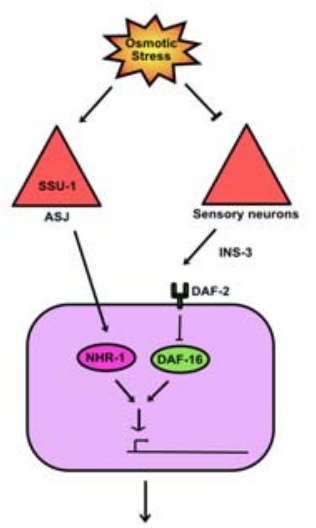

Lysophosphatidylcholine production Developmental arrest 
Figure S1 SSU-1 is not required for DAF-16 translocation into the nucleus in response to osmotic stress. Confocal images of DAF-16::GFP localization after 5 hrs of exposure to $500 \mathrm{mM} \mathrm{NaCl}$ in the wildtype and $s s u-1(f c 73)$ mutants. Scale bars, 10 $\mu \mathrm{m}$. The red dashed lines indicate embryos scored as predominantly nuclear. The white dashed lines indicate embryos scored as predominantly cytoplasmic. Images with a red border were used as representative images in Fig. $3 \mathrm{f}$. 
bioRxiv preprint doi: https://doi.org/10.1101/229393; this version posted December 5, 2017. The copyright holder for this preprint (which was not certified by peer review) is the author/funder, who has granted bioRxiv a license to display the preprint in perpetuity. It is made available under aCC-BY-NC-ND 4.0 International license.

WT $50 \mathrm{mM}$ salt (1/14 predominantly nuclear)

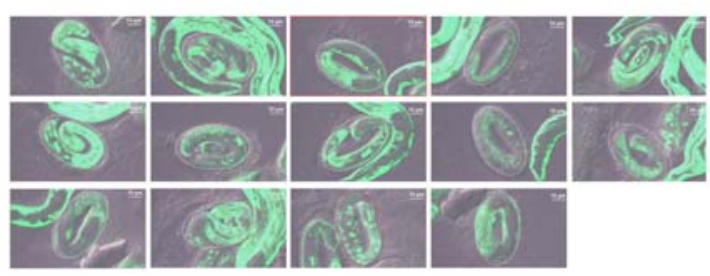

WT $500 \mathrm{mM}$ salt (11/14 predominantly nuclear)
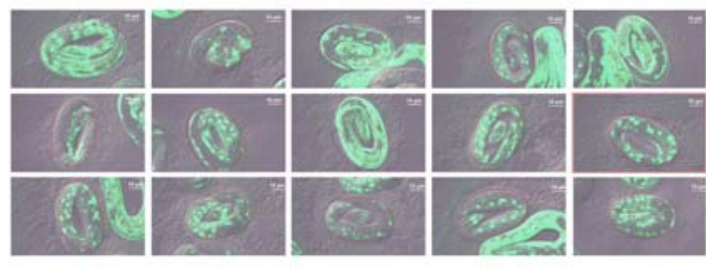

ssu-1(-) 50mM salt (1/15 predomintantly nuclear)
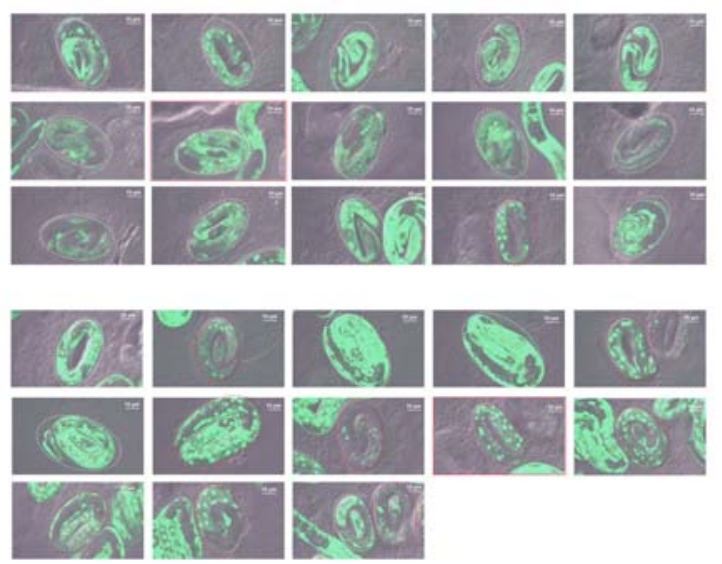

(9/14 predominantly nuclear) 
Supplemental Table 1 Profile of mRNA expression in wild-type and ssu-1(fc73) embryos at $50 \mathrm{mM} \mathrm{NaCl}$ and $500 \mathrm{mM} \mathrm{NaCl}$.

Supplemental Table 2 Profile of mRNA expression in wild-type and daf-16(m26) embryos at $50 \mathrm{mM} \mathrm{NaCl}$ and $500 \mathrm{mM} \mathrm{NaCl}$.

Supplemental Table 3 Profile of lipid and polar metabolites in wild-type, ssu-1(fc73), and daf-16(m26) embryos from parents grown at $50 \mathrm{mM}$ and $300 \mathrm{mM} \mathrm{NaCl}$.

Supplemental Table 4 Statistics source data. 